\title{
Impact of Aortic Arch Anatomy on Technical Performance and Clinical Outcomes in Patients with Acute Ischemic Stroke
}

\author{
(D).A. Knox, (D) M.D. Alexander, (DD.B. McCoy, DD.C. Murph, (DP.J. Hinckley, (D).C. Ch'ang, (D)C.F. Dowd, (D)V.V. Halbach,
}

(D) R.T. Higashida, (D) M.R. Amans, (D) S.W. Hetts, and (DD.L. Cooke

\begin{abstract}
BACKGROUND AND PURPOSE: Arterial access is a technical consideration of mechanical thrombectomy that may affect procedural time, but few studies exist detailing the relationship of anatomy to procedural times and patient outcomes. We sought to investigate the respective impact of aortic arch and carotid artery anatomy on endovascular procedural times in patients with large-vessel occlusion.
\end{abstract}

MATERIALS AND METHODS: We retrospectively reviewed imaging and medical records of 207 patients from 2 academic institutions who underwent mechanical thrombectomy for anterior circulation large-vessel occlusion from January 2015 to July 2018. Preintervention CTAs were assessed to measure features of the aortic arch and ipsilateral great vessel anatomy. These included the cranial-to-caudal distance from the origin of the innominate artery to the top of the aortic arch and the takeoff angle of the respective great vessel from the arch. mRS scores were calculated from rehabilitation and other outpatient documentation. We performed bootstrap, stepwise regressions to model groin puncture to reperfusion time and binary mRS outcomes (good outcome, $m R S \leq 2)$.

RESULTS: From our linear regression for groin puncture to reperfusion time, we found a significant association of the great vessel takeoff angle $(P=.002)$ and caudal distance from the origin of the innominate artery to the top of the aortic arch $(P=.05)$. Regression analysis for the binary $\mathrm{mRS}$ revealed a significant association with groin puncture to reperfusion time $(P<.001)$.

CONCLUSIONS: These results demonstrate that patients with larger takeoff angles and extreme aortic arches have an association with longer procedural times as approached from transfemoral access routes.

ABBREVIATIONS: $\mathrm{CCA}=$ common carotid artery; $\mathrm{CCIA}=$ the cranial-to-caudal distance from the origin of the innominate artery to the top of the aortic arch; GPRT = groin puncture to reperfusion time; HTN = hypertension; LVO = large-vessel occlusion

I schemic stroke is the fifth leading cause of death and the leading cause of long-term disability in the United States. ${ }^{1}$ Recent studies have established mechanical thrombectomy as the criterion standard of care for large-vessel occlusion over tPA alone. ${ }^{2-5}$ These and other studies have demonstrated that reduced time between the onset of symptoms and reperfusion leads to better clinical outcomes. ${ }^{6,7}$ That time interval comprises prehospital

Received July 14, 2019; accepted after revision December 11.

From the Departments of Radiology (J.A.K., D.B.M., D.C.M., P.J.H., C.F.D., V.V.H., R.T.H., M.R.A., S.W.H., D.L.C.) and Neurology (J.C.C.), University of California, San Francisco, San Francisco, California; and Department of Radiology (M.D.A.), University of Utah, Salt Lake City, Utah.

This publication was supported by the National Center for Advancing Translational Sciences, National Institutes of Health, through the University of California, San Francisco-Clinical \& Translational Science Institute grant No. TL1 TR001871.

Please address correspondence to Joseph A. Knox, BA, University of California, San Francisco, Department of Radiology, 513 Parnassus Ave, Suite S-245, San Francisco, CA 94143; e-mail: joseph.knox@ucsf.edu

- Indicates open access to non-subscribers at www.ajnr.org

http://dx.doi.org/10.3174/ajnr.A6422 and in-house elements, but physicians can largely affect only the latter. The interplay of minimizing the time from a patient's symptom onset to reperfusion with the degree of reperfusion itself is at the core of current practice for the treatment of largevessel occlusion (LVO).

While challenging arterial anatomy can lead to longer procedural times, scant literature exists detailing the impact of arch and carotid anatomy on procedural times. Ribo et $\mathrm{al}^{8}$ demonstrated that patients with difficult access have lower rates of recanalization and that carotid artery tortuosity can contribute to difficult access, but the aortic arch and carotid anatomy were not measured in this analysis. Kaymaz et $\mathrm{al}^{9}$ found a significant correlation between internal carotid access time and vessel anatomy, specifically the takeoff angle of the left common carotid artery (CCA)/innominate artery and CCA tortuosity. While these studies demonstrate that carotid tortuosity may extend the time to reperfusion, the impact of aortic arch anatomy on operative times has not been demonstrated. Additionally, no studies have looked 
Table 1: Demographic variables

\begin{tabular}{lc}
\hline \multicolumn{1}{c}{ Variable } & Mean or \% \\
\hline Age (yr) & 69.4 (range, 21-98) \\
Sex (male) & $45.4 \%$ \\
Side (left) & $54.6 \%$ \\
Ethnicity & \\
$\quad$ White & $56.9 \%$ \\
Asian & $21.6 \%$ \\
Hispanic & $13.7 \%$ \\
African American & $7.4 \%$ \\
Pacific Islander & $0.5 \%$ \\
Hypertension & $68.1 \%$ \\
Hyperlipidemia & $44.4 \%$ \\
Diabetes mellitus & $24.2 \%$ \\
Coronary/peripheral artery disease & $24.2 \%$ \\
Atrial fibrillation & $44.9 \%$ \\
Prior stroke & $14.0 \%$ \\
\hline
\end{tabular}

at the impact of the aortic arch and carotid artery tortuosity on clinical outcomes in LVO.

The purpose of this study was to investigate the respective impact of the aortic arch and carotid artery anatomy on endovascular procedural times in patients with LVO and to build a multivariable model that may be prospectively applied to better direct arterial access approaches during LVO intervention.

\section{MATERIALS AND METHODS}

\section{Patient Selection}

This study was institutional review board-approved at both the University of California San Francisco and the University of Utah, and no informed consent was required. A total of 207 consecutive patients who underwent mechanical thrombectomy for anterior circulation LVO from January 2015 to July 2018 were included in this study. We had patient cohorts from 3 separate sites at 3 separate academic centers, which are staffed by fellowship-trained neurointervnetional surgeons. We excluded patients who had an aborted endovascular intervention and acute ischemic stroke in the posterior circulation. Reasons for aborted interventions ranged from collateral filling seen during the intervention to rapid patient decompensation.

Patient and procedural data were collected retrospectively from the electronic medical record. Data points collected included the following: demographics (age at time of thrombectomy, sex, ethnicity), medical history (eg, hypertension [HTN], hyperlipidemia, diabetes mellitus, coronary artery disease/peripheral artery disease, atrial fibrillation, prior stroke), acute ischemic stroke episode details (eg, baseline mRS and NIHSS, last seen healthy to endovascular procedure start time, clot side, clot location, ASPECTS), and procedural intervention details (eg, exchange wire used, Simmons catheter use, stent placement, angioplasty, procedural time). Ethnicity data was collected due to the well-established differences in stroke rates amongst our studied ethnic groups. See Table 1 for basic demographic information on our study cohort.

Our primary outcome measures included groin puncture to reperfusion time (GPRT) and mRS, which were recorded from the medical record at discharge, 30 days, and 90 days. $^{10}$

\section{Procedure}

Surgical procedures were performed by 9 different neurointerventional surgeons, each having at least 5 years of experience in endovascular stroke treatment at quaternary centers. All procedures were performed with the patient under moderate sedation or monitored anesthesia care, with general endotracheal anesthesia used only when necessary. Case selection was as prescribed by the 2015 and later 2018 American Heart Association/American Stroke Association guidelines. Equipment choice and technical details were case-specific as directed by the attending interventionalist at each site. Each procedure was performed according to the purview of the attending surgeon, the details of which were dictated by the clinical condition, though a stent retriever in tandem with catheter aspiration was the most frequently used thrombectomy technique.

\section{Anatomic Measurement Protocol}

CTA images were independently reviewed by 3 board-certified neuroradiologists and 1 trained medical student. Preprocedural CTA images were reviewed in the axial, coronal, and sagittal planes to generate anatomic metrics. Imaging analyses for the parameters of the aortic arch and carotid artery were similar to those described in the literature. ${ }^{9,11}$ Carotid and aortic length and angulation were directly calculated in the sagittal and coronal planes. Carotid and aortic length were defined as the curvilinear distance between the vessel origin and end. We limited our measurements to those that could be quickly generated from a CTA, including aortic arch variables (aortic arch types 1-3, medial-tolateral span, anterior-to-posterior span, and the cranial-to-caudal distance from the origin of the innominate artery to the top of the aortic arch [CCIA]) and carotid artery variables (CCA/innominate takeoff angle, medial-to-lateral span of the CCA, and anterior-to-posterior span of the CCA). For anatomic spans, measurements were made with the caliper extending from the specific dimensional extreme at the margin of the target vessel to the edge of the film as a reference, allowing measurement across multiple cuts. For the takeoff angle, the angle between vessels was defined as the angle of the vessel off the aortic arch in reference to a straight line down the curve of the spine. For right-sided strokes, the innominate angle was calculated, and for left-sided strokes, the CCA angle was calculated. For arch type (I, II, or III), classifications were made as previously described. ${ }^{12}$ Figure 1 gives detailed examples of these measurements and calculations.

\section{Statistical Analysis}

Statistical analyses were performed in R, Version 3.5 (http:// www.r-project.org/). ${ }^{13}$ Before regression analysis, univariate analysis (logistic for mRS binary outcome and linear for reperfusion time) was used to determine which variables were independently associated with each outcome. The variables with $P$ values $<.1$ were included in subsequent stepwise regression modeling. Because the most recent mRS score was limited (90-day followup), a composite variable was created, representing the most recent $\mathrm{mRS}$ score available for each patient. A binary mRS variable was created using the most recent mRS postprocedural metric, which stratified patients into either those with an $\mathrm{mRS}$ score of $\leq 2$ or those with an mRS score of $>2$. 


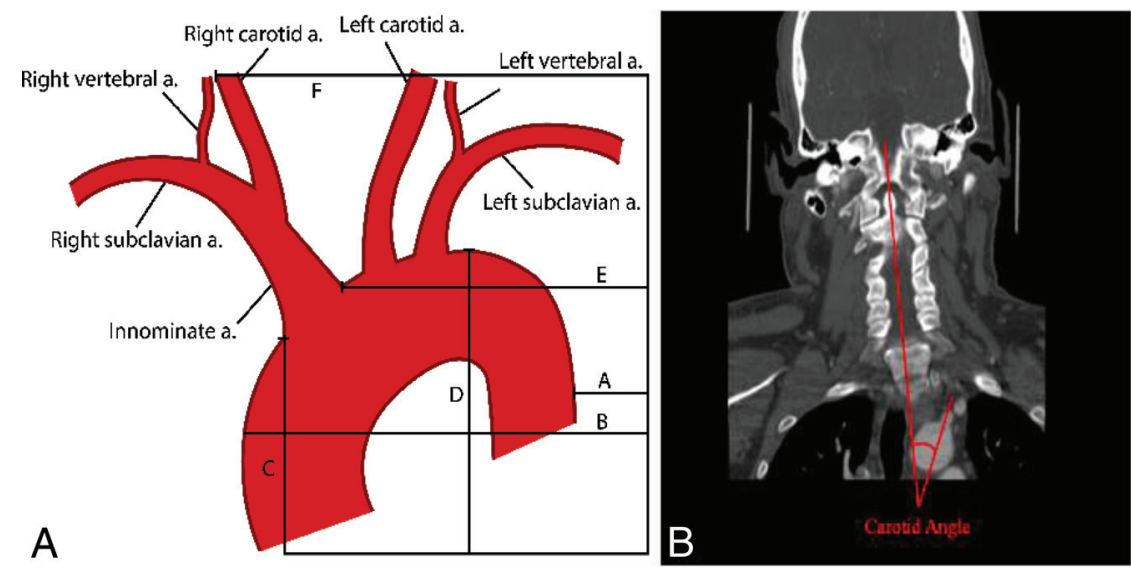

FIG 1. A, Diagram showing the calculation of sample measurements. B minus A represents the medial-to-lateral span of the aorta. $E$ minus $F$ represents the medial-to-lateral span of the right carotid artery. D minus $C$ represents the cranial-to-caudal span of the aortic arch. B, Sample takeoff angle measurement of the left CCA on CTA with the angle of the spine as a reference. a. indicates artery.

Table 2: Results of groin puncture to reperfusion bootstrap while controlling for site ${ }^{a}$

\begin{tabular}{lccc}
\hline \multicolumn{1}{c}{ Variable } & Estimate & Standard Error & $\boldsymbol{P}$ Value \\
\hline Simmons (none) & 13.74 & 8.01 & .09 \\
Tandem (none) & $25.65^{\mathrm{b}}$ & $8.0^{\mathrm{b}}$ & $.02^{\mathrm{b}}$ \\
Takeoff angle [10 degrees] & $5.29^{\mathrm{b}}$ & $1.70^{\mathrm{b}}$ & $.002^{\mathrm{b}}$ \\
Aorta cranial-caudal [5 mm] & $2.98^{\mathrm{b}}$ & $1.48^{\mathrm{b}}$ & $.05^{\mathrm{b}}$ \\
Left side (right) & 2.32 & 5.01 & .64 \\
Site 2 (site 1) & 2.48 & 6.42 & .70 \\
Site 3 (site 1) & -12.16 & 6.38 & .06 \\
\hline
\end{tabular}

${ }^{a}$ Categoric values are indicated by parentheses with the baseline comparison within the parentheses. For continuous variables, units are shown in brackets.

${ }^{\mathrm{b}}$ Significant associations.

For both the GPRT outcome and the mRS outcome variable, a bootstrapped, stepwise linear regression model was used to model predictor variables. To determine the predictive validity of each model, we split the 207 observations into a training group (70\%, 145 observations) and testing data (30\%, 62 observations). Mean squared error as a percentage of the GPRT range and accuracy to classify binarized $\mathrm{mRS}$ change was used to determine the predictability of the 2 outcomes. One final model was determined for each outcome on the basis of the frequency of the variables used in the bootstrap and the reliability of the coefficients. In addition, the effect of a triple interaction of the takeoff angle, HTN and age on the GPRT was modeled. The GPRT was thought to be modulated by HTN and age on the basis of clinical experience from neurointerventionalists.

\section{RESULTS}

\section{Anatomic Measurements}

All except 7 patients (3.3\%) had a complete series of images allowing accurate measurement of all anatomic variables. The most common reason for measurements not being obtained was the inferior aspect of images not including the aortic arch. The mean cranial-caudal span of the aorta was $10.9 \pm 8.9 \mathrm{~mm}$. The mean medial-lateral span and anterior-posterior span of the aorta were $61.6 \pm 15.5 \mathrm{~mm}$ and $101.0 \pm 17 \mathrm{~mm}$, respectively. The mean medial-lateral span and anterior-posterior span of the carotid arteries on the affected side were $36.5 \pm 12.4 \mathrm{~mm}$ and $28.2 \pm 14.4 \mathrm{~mm}$, respectively. The mean takeoff angle of either the innominate artery or carotid artery on the affected side was $20.3^{\circ} \pm 16.6^{\circ}$.

\section{Procedural Outcomes}

The mean GPRT was $55.6 \pm 35.2$ minutes with an interquartile range of 30-68 minutes. TICI $2 b-3$ recanalization was achieved in 159 cases (76.8\%), while 12 cases resulted in TICI 0 . One hundred nineteen clots were in M1 (57.5\%), 48 (23.2\%) were in the ICA, $39(18.8 \%)$ were in M2, and 1 case $(0.5 \%)$ was in the CCA. Tandem lesions occurred in 21 cases (10.1\%). Stents or percutaneous transluminal angioplasty was used in $14(6.8 \%)$ and 21 (10.1\%) cases, respectively. A Simmons catheter or an exchange wire was used in 24 (11.6\%) and 48 (23.2\%) cases, respectively. Last seen healthy to groin puncture time and last seen healthy to reperfusion time were found to be highly correlated (Pearson $=0.99$ ); therefore, last seen healthy to reperfusion time was removed from downstream analysis.

For GPRT, the takeoff angle was the strongest and most consistent predictor, being included in $93.9 \%$ of the bootstrap models with coefficients being $100 \%$ positive. Table 2 outlines the results of the final model determined by bootstrap, stepwise regression modeling for the GPRT. The variables included in the final model were Simmons catheter use, tandem lesions, takeoff angle, cranialto-caudal span of the aortic arch, side, and site. Variables that showed individual statistical significance with the GPRT were tandem lesions (tandem lesions resulted in a 25 minute 39 second increase in the GPRT, $P=0.02$ ), takeoff angle (a $10^{\circ}$ angle increase corresponding to a 5 minute 17 second increase in the GPRT, $P=$ .002 ), and CCIA (a 5-mm increase in the CCIA span corresponded to a 2 minute 59 second increase in GPRT, $P=.05$ ). Site 3 compared with site 1 showed a borderline significant decrease in reperfusion times $(-12$ minute 36 second, $P=.06)$. When we applied this model to the test set, the mean squared error was 34 and represents the mean error in minutes. Therefore, our model is accurate within 34 minutes in predicting reperfusion times.

Due to a limited sample size, we were able to find only a borderline significant triple interaction for hypertension, age, and the takeoff angle on the GPRT modeling, though this trending effect is apparent and shown in Fig 2. This interaction modeling demonstrates a shift in the association between the takeoff angle and GPRT as age increases in patients without hypertension $(P=.22)$, which is absent in patients with hypertension (Fig 2). For example, in patients without hypertension, we found that a 1 -SD increase of the takeoff angle was associated with a $16 \mathrm{mi}$ nute 46 second increase in the groin puncture to reperfusion time, whereas for patients with hypertension, a 1-SD increase in 


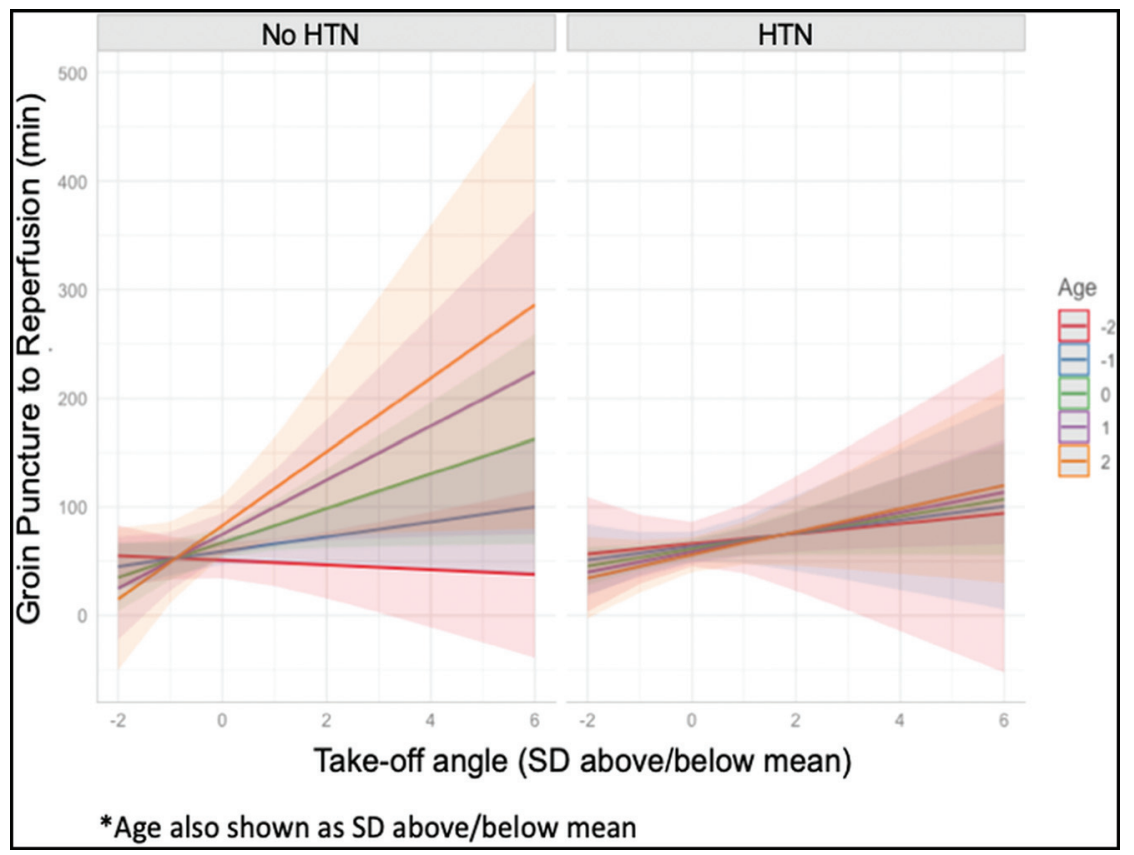

FIG 2. Results of interaction modeling for groin puncture to reperfusion. Associations between the takeoff angle ( $x$-axis) and GPRT in minutes (y-axis) are shown in patients with and without hypertension. Data are standardized to units of the SD from the mean of both age and the takeoff angle. Age is plotted by SDs from the mean from -2 to +2 SDs of age.

Table 3: Results of bootstrap, stepwise logistic regression for binary mRS, controlling for mRS time point ${ }^{\mathrm{a}}$

\begin{tabular}{llccc}
\hline \multicolumn{1}{c}{ Variable } & OR & Lower Cl & Upper Cl & $P$ Value \\
\hline mRS: 30 days (discharge) & $0.16^{\mathrm{b}}$ & $0.04^{\mathrm{b}}$ & $0.54^{\mathrm{b}}$ & $.004^{\mathrm{b}}$ \\
mRS: 90 days (discharge) & $0.13^{\mathrm{b}}$ & $0.04^{\mathrm{b}}$ & $0.40^{\mathrm{b}}$ & $<.001^{\mathrm{b}}$ \\
mRS preprocedure [mRS score] & $3.59^{\mathrm{b}}$ & $2.16^{\mathrm{b}}$ & $6.53^{\mathrm{b}}$ & $<.001^{\mathrm{b}}$ \\
Exchange wire (none) & 2.98 & 1.02 & 9.26 & .05 \\
ASPECTS [ASPECTS] & 0.77 & 0.59 & 1.00 & .05 \\
HTN (none) & $3.08^{\mathrm{b}}$ & $1.08^{\mathrm{b}}$ & $9.30^{\mathrm{b}}$ & $.04^{\mathrm{b}}$ \\
Arch II (I) & 0.57 & 0.19 & 1.65 & .30 \\
Arch III (I) & $1.16^{\mathrm{b}}$ & 0.26 & 5.18 & .85 \\
Site 2 (1) & 1.63 & 0.54 & 5.05 & .39 \\
Site 3 (1) & 0.79 & 0.24 & 2.65 & .70 \\
LSNRT [60 min] & 1.07 & 0.99 & 1.17 & .09 \\
GPRT [15 min] & $1.93^{\mathrm{b}}$ & $1.52^{\mathrm{b}}$ & $2.55^{\mathrm{b}}$ & $<.001^{\mathrm{b}}$ \\
Age [yr] & 0.99 & 0.95 & 1.02 & .47 \\
Aorta medial-lateral [mm] & 1.00 & 0.97 & 1.02 & .82 \\
Carotid anterior-posterior [min] & 1.02 & 0.99 & 1.05 & .16 \\
Side L (R) & 0.46 & 0.18 & 1.09 & .09
\end{tabular}

Note:-LSNRT indicates last seen healthy to reperfusion time; L, left; R, right.

${ }^{a}$ Categoric values are indicated by parentheses with the baseline comparison within the parentheses. For continuous variables, units are shown in brackets.

${ }^{\mathrm{b}}$ Significant associations.

the takeoff angle was associated with a 9 minute 13 second increase in the groin puncture to reperfusion time, all while holding age at an mean value.

\section{Clinical Outcomes}

The mean last seen healthy to reperfusion time was $434.5 \pm 321$ minutes, with an interquartile range of 230-509 minutes. The mean preprocedural and postprocedural NIHSS scores were $16.8 \pm 6.6$ and $10.6 \pm 9.1$, respectively, with 131 (63.3\%) patients having a recorded postprocedural NIHSS. Two-hundred patients
(96.6\%) had a recorded preprocedural mRS, and 187 patients (90.3\%) had an mRS score recorded at discharge. Only $103(49.8 \%)$ and $97(46.9 \%)$ patients had 30-day mRS scores and 90-day mRS scores, respectively. One hundred twenty-five patients $(60.3 \%)$ had either a 30-day or 90-day mRS score available. When patients were dichotomized into mRS $\leq 2$ or $>2$ by the most recent score, $73.8 \%$ were in the higher mRS group, indicating a poor outcome.

Table 3 summarizes the results of the final model determined by bootstrap, stepwise logistic regression for binary mRS outcomes. Statistically significant independent predictors of more severe disability $(\mathrm{mRS}>2)$ included the mRS type (compared with the mRS score recorded at discharge, $\mathrm{mRS}$ at 30 days had a reduced risk of severe $\mathrm{mRS}$ score, $\mathrm{OR}=0.16, P=$ .004 , and similarly for mRS recorded at 90 days, $\mathrm{OR}=0.13, P<.001)$, preprocedural mRS (a 1-unit increase in the preprocedural mRS was associated with a $359 \%$ higher odds of a severe postsurgical score, $P=$ $<.001$ ), hypertension (having hypertension increased the odds of a severe mRS score by 3.08 -fold, $P=.04$ ), and GPRT (a 15-minute increase in the GPRT resulted in a 1.93-times increase of a severe mRS score, $P=$ $<.001)$. A borderline significant variable was the use of an exchange wire (2.98-time increase in a severe mRS score, $P=.05$ ), and a 1-unit increase in the ASPECTS was associated with reduced risk $(\mathrm{OR}=0.77$, $P=.05)$. When applying this model to the test set, we found an accuracy of 80.63 .

\section{DISCUSSION}

Patient demographic data, outcomes, and procedural results in our cohort were comparable with the populations and results of large-scale mechanical thrombectomy studies. ${ }^{5,14}$ Notably, our cohort had higher rates of atrial fibrillation at $45 \%$. In addition, despite the higher last seen healthy to reperfusion time of our cohort compared with other studies, our outcome data are similar, with $73 \%$ of patients in the $\mathrm{mRS}>2$ group for the most recent $\mathrm{mRS}$ score. The longer last seen healthy to reperfusion time is likely because we did not include a temporal cutoff from stroke onset to presentation as previous studies did. 
From the measured anatomic variables, we found that the takeoff angle and CCIA were significant independent predictors of procedural time. Although the CCIA has never been specifically shown to have an influence on procedural times in mechanical thrombectomy, unfavorable arch types (type II or III) have been associated with more ischemic events and longer procedural times in patients undergoing carotid stent placement. ${ }^{15-18}$ However, most of these studies found subjective associations primarily with arch type or, in 1 study, a composite "B.A.D. score" based on bovine variation, arotic arch type, and ICA dolichoarteriopathy. ${ }^{18}$ The 1 study to directly measure angulation did not use groin puncture to reperfusion time or clinical status as outcome measures but rather fluoroscopy time. ${ }^{19}$ In addition, all of these studies were from a single institution, with only 1 study including both procedural time measures and clinical outcomes. While we did not find arch type itself to be predictive of procedural time as in prior studies, the CCIA can be seen as a linear variable transformation of the ordinal variable arch type. ${ }^{12}$ Among the 2 anatomic predictors of procedural time, the takeoff angle was more statistically significant and had a larger range of values. One prior study on mechanical thrombectomy found the takeoff angle of the CCA and the innominate artery as well as the tortuosity of the CCA to have the most significant influence in internal carotid artery access time, in agreement with our results. ${ }^{9}$ Collectively, these results confirm the notion that the takeoff angle of the innominate artery or left CCA is the first and possibly most difficult hurdle in ICA access from a femoral approach. Notably, our study examined overall procedural time and included a multivariate analysis compared with examination of internal carotid access time by univariate analysis of the prior study. The anatomic factors that we examined were chosen on the basis of calculations that could be standardized and quickly performed on preprocedural CTAs; thus, tortuosity was not included as a variable due to its more involved measurement criteria. ${ }^{9}$ The measurement of the takeoff angle in reference to the spine can likely be accomplished in $<30$ seconds by an experienced user, emphasizing the relative brevity of selected measurements in an emergency LVO.

When examining the effects of age and hypertension on the takeoff angle and procedural times through triple-interaction modeling, we found age to be a modifier of the effect of the takeoff angle on procedural times in patients without hypertension. The tortuosity of the carotid arteries seen in older patients appears to be influenced by long-standing hypertension and atherosclerosis. $^{20-22}$ Older patients without hypertension appear to be the most difficult cohort to access from our analysis, suggesting another etiology to the apparent tortuosity in the patient population, such as atherosclerosis. Thus, the presence or absence of HTN as a function of age conveys properties about the rigidity of the vessel wall, a dimension we have less success in capturing through conventional imaging alone. ${ }^{23}$ Overall, our results suggest that an operator with a few pieces of information such as the takeoff angle, HTN status, and age may be able to make better judgments around a case with the ultimate goal of shrinking time to reperfusion. ${ }^{24}$

From our clinical outcome analysis, we found that faster procedural times and last seen healthy to reperfusion times were associated with better outcomes, in agreement with prior studies. ${ }^{25,26}$ Procedural times did significantly vary by site, but this was controlled for in our logistic regression. We did not find last seen healthy to reperfusion time to be significantly associated with more severe disability; however, it trended toward significance. This trend is likely due to the subjective nature of this variable, in addition to limitations of data collection from our county hospital site. We also did not find the ASPECTS to be a significant predictor of more severe disability, but this finding was likely due to the relative distribution of our ASPECTS with $>90 \%$ being $\geq 6$.

On the basis of the results, operators may consider the use of a Simmons catheter at case start for instances with a carotid takeoff angle of $>10^{\circ}$ and/or an innominate origin of $5 \mathrm{~mm}$ caudal to the aortic apex. Furthermore, if a Simmons catheter is ineffective after 10 minutes of effort, operators should consider an alternative access approach (eg, carotid or radial), depending on their level of comfort and the particular clinical scenario. If a transcervical approach is used, general anesthesia is recommended; thus, mean arterial pressures must be maintained during induction and throughout the anesthesia period. In these cases, at our institution, arterial closure is performed by vascular or neurologic surgery, though other methods (eg, manual compression, closure device) have been described. The authors have no experience with transradial approaches for stroke, though we are aware of series describing success in both the anterior and posterior circulations. ${ }^{27,28}$ Prospective study of patients with anatomy that may prove more difficult to access via a transfemoral route using such alternative methods would benefit the community.

The retrospective nature of this study is a limitation. In addition, a certain amount of error should be taken into account for the measurement of takeoff angles, because this is the most subjective of our anatomic variables. For our clinical outcome analysis, we did not have access to longitudinal outcome data for half of our patients and thus had to create a composite mRS score for all patients. We did control for the time point in our logistic regression analysis to try to overcome this limitation. Finally, because this was a multi-institutional study, there were likely unstudied technical differences among institutions.

\section{CONCLUSIONS}

There was a strong association between larger takeoff angles and higher aortic arches and longer procedural times, especially in older patients without hypertension. This study presents a unique, comprehensive analysis of objective measurements of aortic arch anatomic factors, examining their effect on both procedural times and clinical outcomes. A nonfemoral access method such as direct carotid puncture should be considered when one is presented with these difficult patient and anatomic factors, to minimize procedural duration.

\section{ACKNOWLEDGMENTS}

We would like to thank Jacquelyn Withers for helping design our figures.

Disclosures: Joseph A. Knox-RELATED: Grant: National Institutes of Health TL1 TR001871, Comments: I received a National Institutes of Health TL1 research grant to perform yearlong research at the University of California, 
San Francisco, where I attend the medical school. This research was supported by that grant. Christopher F. Dowd-UNRELATED: Other: MicroVention, Comments: Chief Adjudicator for the FRED Flow Diverter Clinical Trial.* Matthew R. Amans-UNRELATED: Consultancy: Medtronic, Comments: Pipeline proctor; Grants/Grants Pending: National Institutes of Health, Comments: National Institutes of Health R21DC016087. *Money paid to the institution.

\section{REFERENCES}

1. Benjamin EJ, Blaha MJ, Chiuve SE, et al; American Heart Association Statistics Committee and Stroke Statistics Subcommittee. Heart Disease and Stroke Statistics: 2017 Update-A Report from the American Heart Association. Circulation 2017;135:e146-603 CrossRef Medline

2. Saver JL, Goyal M, Bonafe A, et al; SWIFT PRIME Investigators. Stent-retriever thrombectomy after intravenous t-PA vs. t-PA alone in stroke. $N$ Engl J Med 2015;372:2285-95 CrossRef Medline

3. Goyal M, Demchuk AM, Menon BK, et al; ESCAPE Trial Investigators. Randomized assessment of rapid endovascular treatment of ischemic stroke. $N$ Engl J Med 2015;372:1019-30 CrossRef Medline

4. Campbell BC, Mitchell PJ, Kleinig TJ, et al. Endovascular therapy for ischemic stroke with perfusion-imaging selection. $N$ Engl J Med 2015;372:1009-18 CrossRef Medline

5. Berkhemer OA, Fransen PS, Beumer D, et al; MR CLEAN Investigators. A randomized trial of intraarterial treatment for acute ischemic stroke. $N$ Engl J Med 2015;372:11-20 CrossRef Medline

6. Hassan AE, Chaudhry SA, Miley JT, et al. Microcatheter to recanalization (procedure time) predicts outcomes in endovascular treatment in patients with acute ischemic stroke: when do we stop? AJNR Am J Neuroradiol 2013;34:354-59 CrossRef Medline

7. Spiotta AM, Vargas J, Turner R, et al. The golden hour of stroke intervention: effect of thrombectomy procedural time in acute ischemic stroke on outcome. J Neurointerv Surg 2014;6:511-16 CrossRef Medline

8. Ribo M, Flores A, Rubiera M, et al. Difficult catheter access to the occluded vessel during endovascular treatment of acute ischemic stroke is associated with worse clinical outcome. J Neurointerv Surg 2013;5(Suppl 1):170-73 CrossRef Medline

9. Kaymaz Z, Nikoubashman O, Brockmann M, et al. Influence of carotid tortuosity on internal carotid artery access time in the treatment of acute ischemic stroke. Interv Neuroradiol 2017;23:583-88 CrossRef

10. Banks JL, Marotta CA. Outcomes validity and reliability of the Modified Rankin Scale: implications for stroke clinical trials: a literature review and synthesis. Stroke 2007;38:1091-96 CrossRef Medline

11. Boufi M, Guivier-Curien C, Loundou AD, et al. Morphological analysis of healthy aortic arch. Eur J Vasc Endovasc Surg 2017;53:663-70 CrossRef Medline

12. Lin SC, Trocciola SM, Rhee J, et al. Analysis of anatomic factors and age in patients undergoing carotid angioplasty and stenting. Ann Vasc Surg 2005;19:798-804 Medline

13. R Foundation for Statistical Computing. R: A Language and Environment for Statistical Computing, 2018. https://www.R-project.org. Accessed January 31, 2019
14. Campbell BC, Mitchell PJ, Kleinig TJ, et al; EXTEND-IA Investigators. Endovascular therapy for ischemic stroke with perfusionimaging selection. $N$ Engl J Med 2015;372:1009-18 CrossRef Medline

15. Dumont TM, Mokin M, Wach MM, et al. Understanding risk factors for perioperative ischemic events with carotid stenting: is patient age over 80 years or is unfavorable arch anatomy to blame? J Neurointerv Surg 2014;6:219-24 CrossRef Medline

16. Burzotta F, Nerla R, Pirozzolo G, et al. Clinical and procedural impact of aortic arch anatomic variants in carotid stenting procedures. Catheter Cardiovasc Interv 2015;86:480-89 CrossRef Medline

17. Müller MD, Ahlhelm FJ, Von Hessling A, et al. Vascular anatomy predicts the risk of cerebral ischemia in patients randomized to carotid stenting versus endarterectomy. Stroke 2017;48:1285-92 CrossRef Medline

18. Snelling BM, Sur S, Shah SS, et al. Unfavorable vascular anatomy is associated with increased revascularization time and worse outcome in anterior circulation thrombectomy. World Neurosurg 2018;120:e976-83 CrossRef Medline

19. Madhwal S, Rajagopal V, Bhatt DL, et al. Predictors of difficult carotid stenting as determined by aortic arch angiography. I Invasive Cardiol 2008;20:200-04 Medline

20. Pancera P, Ribul M, Presciuttini B, et al. Prevalence of carotid artery kinking in 590 consecutive subjects evaluated by Echocolordoppler: is there a correlation with arterial hypertension? J Intern Med 2000;248:7-12 CrossRef

21. Del Corso L, Moruzzo D, Conte B, et al. Tortuosity, kinking, and coiling of the carotid artery: expression of atherosclerosis or aging? Angiology 1998;49:361-71 CrossRef Medline

22. Han HC. Twisted blood vessels: symptoms, etiology and biomechanical mechanisms. J Vasc Res 2012;49:185-87 CrossRef Medline

23. Messas E, Pernot M, Couade M. Arterial wall elasticity: state of the art and future prospects. Diagn Interv Imaging 2013;94:561-69 CrossRef Medline

24. Bourcier R, Goyal M, Liebeskind DS, et al; HERMES Trialists Collaboration. Association of time from stroke onset to groin puncture with quality of reperfusion after mechanical thrombectomy: a meta-analysis of individual patient data from 7 randomized clinical trials. JAMA Neurol 2019;76:405-11 CrossRef Medline

25. Schmitz ML, Yeatts SD, Tomsick TA, et al. Recanalization and angiographic reperfusion are both associated with a favorable clinical outcome in the IMS III trial. Interv Neurol 2016;5:118-12 CrossRef Medline

26. Mulder M, Jansen IG, Goldhoorn RJ, et al. Time to endovascular treatment and outcome in acute ischemic stroke: MR CLEAN Registry results. Circulation 2018;138:232-40 CrossRef Medline

27. Chen SH, Snelling BM, Sur S, et al. Transradial versus transfemoral access for anterior circulation mechanical thrombectomy: comparison of technical and clinical outcomes. J Neurointervent Surg 2019;11:874-78 CrossRef Medline

28. Maud A, Khatri R, Chaudhry MRA, et al. Transradial access results in faster skin puncture to reperfusion time than transfemoral access in posterior circulation mechanical thrombectomy. J Vasc Interv Neurol 2019;10:53-57 Medline 